Scheele D, Kendrick KM, Khouri C, Kretzer E, Stoffel-Wagner B, Güntürkün $O$ et al (2014). An oxytocin-induced facilitation of neural and emotional responses to social touch correlates inversely with autism traits. Neuropsychopharmacology 39 2078-2085.

Striepens N, Kendrick KM, Hanking V, Landgraf R, Wuellner U, Maier W et al (2013). Elevated cerebrospinal fluid and blood concentrations of oxytocin following its intranasal administration in humans. Sci Rep 3: 3440 .

Neuropsychopharmacology Reviews (2017) 42, 377-378. doi: I 0.1 038/npp.2016.188

\section{A Self-Generated Environmental Factor as a Potential Contributor to Atypical Early Social Communication in Autism}

Autism spectrum disorder (ASD) is defined by deficits in reciprocal social communication and interactions, as well as deficits in cognitive and behavioral flexibility. In the United States, the mean age for diagnosis of ASD is 4; diagnosis can be made as early as age 2 . There is an intense interest in identifying much earlier signs of ASD because of the proven effectiveness of early intervention. Many infant behaviors, such as atypical cries (Esposito and Venuti, 2010), are being explored as such signs. However, the manner in which such early signs of ASD are causally involved in the developmental trajectory of cardinal symptoms of ASD remains unclear.

This picture is about to change, thanks to the development of mouse models of highly reliable genetic risk factors. Many cases of duplications or hemizygous deletions of kilo- to megabase chromosomal segments, termed copy number variants (CNVs), are robustly and reproducibly associated with high rates of ASD. For example, it has been known since 2001 that ASD is seen at high rates among carriers of 22q11.2 hemizygosity (Hiroi et al,
2013). Since 2007, other human CNVs have been similarly found to be associated with high rates of ASD.

Our group has altered copy numbers of small chromosomal segments within the 22q11.2 CNV and identified Tbx1 as one of the 22q11.2 genes critical for various signs of ASD (Hiramoto et al, 2011; Hiroi et al, 2013). Moreover, we have developed and tested an experimental procedure to reliably assess the effects of typical and atypical vocal call sequences on maternal behavior. Our data showed that Tbx1 heterozygosity caused atypical pup vocal call sequences, which then evoked less than optimal maternal care (Takahashi et al, 2016).

Less than optimal maternal care can be considered a 'self-generated environmental factor' of ASD, as it is induced, through atypical vocal sequences, by a genetic ASD risk carrier. Such an environmental factor clearly differs from those that unilaterally impact-and are passively perceived by-risk carriers, such as accidental environmental insults (eg, fetal and infant exposures to pesticides, viruses, and chemicals). The negative phenotypic loop between a risk carrier and its mother is likely to affect the developmental trajectory of ASD symptoms. Certainly, such a hypothesis is consistent with empirical evidence that parent-mediated interventions are effective in alleviating the ultimate degree of some ASD symptoms in humans (Green et al, 2010; Wetherby et al, 2014).

The Refrigerator Mother theory of autism claimed that autism was caused by a lack of maternal warmth. Our view does not attribute the causative event of ASD to mothers.

We submit that less than optimal maternal care is caused by atypical vocal signals of carriers of genetic risk factors and has a modulatory-not causative-impact on the developmental trajectory of severity of ASD symptoms. Our mouse-based hypothesis provides a novel potential mechanistic basis to improve our understanding of the developmental trajectory of ASD and innovative theoretical grounds to develop effective therapeutic interventions.

\section{FUNDING AND DISCLOSURE}

This work was supported by the NIH (R01MH099660) to NH. NH declares that he has received a research grant from Astellas Pharma Inc. TK declares no financial conflict of interest.

\section{ACKNOWLEDGMENTS}

We thank members of the Kikusui and Hiroi laboratories for comments.

\section{Takefumi Kikusui ${ }^{1}$ and Noboru Hiroi ${ }^{2,3,4}$}

${ }^{1}$ Department of Animal Science and Biotechnology, Azabu University, Sagamihara, Japan; ${ }^{2}$ Department of Psychiatry and Behavioral Sciences, Albert Einstein College of Medicine, Bronx, NY, USA; ${ }^{3}$ Dominick P. Purpura Department of Neuroscience, Albert Einstein College of Medicine, Bronx, NY, USA; ${ }^{4}$ Department of Genetics, Albert Einstein College of Medicine, Bronx, NY, USA

E-mail: noboru.hiroi@einstein.yu.edu

Esposito G, Venuti P (2010). Developmental changes in the fundamental frequency (fO) of infants' cries: a study of children with autism spectrum disorder. Early Child Dev Care 180: 1093-1102.

Green J, Charman T, McConachie H, Aldred C, Slonims V, Howlin P et al (2010). Parent-mediated communication-focused treatment in children with autism (PACT): a randomised controlled trial. Lancet 375: 2152-2160.

Hiramoto T, Kang G, Suzuki G, Satoh Y, Kucherlapati R, Watanabe $Y$ et al (2011). Tbx1: identification of a 22 q11.2 gene as a risk factor for autism spectrum disorder in a mouse model. Hum Mol Genet 20: 4775-4785.

Hiroi N, Takahashi T, Hishimoto A, Izumi T, Boku S, Hiramoto T (2013). Copy number variation at 22q11.2: from rare variants to common mechanisms of developmental neuropsychiatric disorders. Mol Psychiatry 18: 1153-1165.

Takahashi T, Okabe S, O'Broin P, Nishi A, Ye K, Beckert $\mathrm{M}$ et al (2016). Structure and function of neonatal social communication in a genetic mouse model of autism. Mol Psychiatry 21: 1208-1214.

Wetherby AM, Guthrie W, Woods J, Schatschneider C, Holland RD, Morgan L et al (2014). Parentimplemented social intervention for toddlers with autism: an RCT. Pediatrics 134: 1084-1093.

Neuropsychopharmacology Reviews (2017) 42, 378; doi: I 0. I 038/npp.2016.225 